\title{
Towards a new set of classification criteria for PFAPA syndrome
}

\author{
Federica Vanoni ${ }^{1,2^{*}}$ (1) , Roberta Caorsi ${ }^{3 \dagger}$, Sandra Aeby ${ }^{2}$, Marie Cochard ${ }^{2}$, Jordi Antón ${ }^{4}$, Stefan Berg ${ }^{5}$, Riva Brik ${ }^{6}$, \\ Pavla Dolezalova ${ }^{7}$, Isabelle Koné-Paut ${ }^{8}$, Benedicte Neven ${ }^{9}$, Seza Ozen ${ }^{10}$, Pascal Pillet ${ }^{11}$, Silvia Stojanov ${ }^{12}$, \\ Carine Wouters $^{13}$, Marco Gattorno ${ }^{3 \dagger}$ and Michaël Hofer ${ }^{2+}$
}

\begin{abstract}
Background: Diagnosis of Periodic Fever, Aphthous stomatitis, Pharyngitis and Cervical Adenitis (PFAPA) syndrome is currently based on the modified Marshall's criteria, but no validated evidence based classification criteria for PFAPA has been established so far.

Methods: A multistep process, based on the Delphi and Nominal Group Technique was conducted. After 2 rounds of e-mail Delphi survey involving 21 experts in autoinflammation we obtained a list of variables that were discussed in an International Consensus Conference. Variables reaching the $80 \%$ of consensus between participants were included in the new classification criteria.

In the second phase the new classification criteria and the modified Marshall's criteria were applied on a cohort of 80 pediatric PFAPA patients to compare their performance.

Results: The Delphi Survey was sent to 22 participants, 21 accepted to participate. Thirty variables were obtained from the survey and have been discussed at the Consensus Conference. Through the Nominal Group Technique we obtained a new set of classification criteria. These criteria were more restrictive in respect to the modified Marshall's criteria when applied on our cohort of patients.

Conclusion: Our work led us to identify a new set of classification criteria for PFAPA syndrome, but they resulted to be too restrictive to be applied in daily clinical practice for the diagnosis of PFAPA.
\end{abstract}

Keywords: Autoinflammatory diseases, PFAPA

\section{Background}

Periodic Fever, Aphthous stomatitis, Pharyngitis and Cervical Adenitis (PFAPA) syndrome is characterized by regularly recurrent fever flares of early onset, accompanied by pharyngitis, cervical lymphadenopathy and oral aphthous ulcers [1]. The diagnosis is currently based on the modified Marshall's criteria proposed in 1999 [2], but the power of these criteria remains limited, and they show a good sensitivity but a lack in specificity [3]. PFAPA is not a well-defined disease and shows a clinical overlap with the

\footnotetext{
* Correspondence: federica.vanoni@eoc.ch

${ }^{\dagger}$ Federica Vanoni, Roberta Caorsi, Marco Gattorno and Michaël Hofer contributed equally to this work.

${ }^{1}$ Istituto Pediatrico della Svizzera Italiana, Ospedale San Giovanni, 6500 Bellinzona, Switzerland

${ }^{2}$ Unité Romande d'Immuno-rhumatologie Pédiatrique, CHUV, University of Lausanne, Lausanne and HUG, Geneva, Switzerland

Full list of author information is available at the end of the article
}

inherited periodic fevers, such as Familial Mediterranean Fever, Tumor necrosis factor receptor-associated periodic syndrome and Mevalonate kinase deficiency, for which a causative gene is well established. These diseases often require a more intensive therapeutic approach and have a more severe course and long-term prognosis. Therefore among those showing a PFAPA phenotype, it would be important to identify patients at risk of carrying mutation in genes associated with inherited periodic fever to avoid unnecessary genetic testing [3]. Moreover, PFAPA shows a clinical overlap with other infectious diseases, and more specific criteria could help avoid prescriptions of unnecessary antibiotic therapies.

In 2008 we started an international collaborative effort, aimed at developing new classification criteria based on expert consensus and analysis of real patient data, to identify a new set of classification criteria for PFAPA

(C) The Author(s). 2018 Open Access This article is distributed under the terms of the Creative Commons Attribution 4.0 International License (http://creativecommons.org/licenses/by/4.0/), which permits unrestricted use, distribution, and 
syndrome with higher specificity. This project was conducted through several steps: 1) a preliminary Delphi survey among international pediatricians and rheumatologists; 2) a consensus conference of physicians with specific expertise in PFAPA to obtain a provisional new set of classification criteria; 3 ) a preliminary evaluation of the new set of criteria on a cohort of real PFAPA patients.

\section{Methods}

At first we proceeded to the creation of the classification criteria by using the Delphi and Nominal Group Technique [4]. We performed 2 rounds of e-mail Delphi survey involving 22 experts in autoinflammation to propose criteria for the diagnosis of PFAPA: first the experts listed the most relevant variables in their opinion for the diagnosis of PFAPA, and the proposed variables were sorted in 8 categories; secondly, the experts had to validate categories and individual items by groups of symptoms ranking the criteria. Variables coming from this survey were discussed in an International Conference that was held in Morges, Switzerland, in November 2008, with 11 experts. By using the Nominal Group technique, each variable included in the list was discussed and included in the classification criteria if a consensus of $80 \%$ was reached.

In the second phase we aimed to evaluate the new set of criteria on a group of patients with a diagnosis of PFAPA given at a specialized clinic for pediatric rheumatology. Thus, we applied the new set of classification criteria and the modified Marshall's criteria on a cohort of 80 pediatric PFAPA patients from Lausanne (Switzerland) and Genoa (Italy) pediatric rheumatology units to compare their performance. For this study we selected all the patients with a diagnosis of PFAPA validated by an experienced pediatric rheumatologist ( $\mathrm{MH}$ and $\mathrm{MG}$ ), with complete information on variables included in both sets of criteria.

\section{Results}

The Delphi Survey was sent to 22 participants, 21 accepted to participate, one didn't respond.

Seventy-two different variables were obtained from the first survey, and could be sorted in 8 different categories. After the second Delphi, 6 categories reached consensus, and 2 individual variables; the other categories and variables have been discussed at the Consensus Conference (Table 1).

Through the Nominal Group Technique we obtained a new set of classification.

criteria (Table 2).

Different version proposed for some variables were ranked by participants to choose the most appropriated one (e.g. duration of attacks: $>2$ days, $>3$ days, $<5$ days, 3-6 days, max 5 days, or max 6 days).

Periodicity of the febrile episodes as well as the maximum interval between episodes were specified in the new
Table 1 Categories and variables reaching consensus during both Delphi

\begin{tabular}{ll}
\hline Categories/variables & Consensus (80\%) \\
\hline 1. Recurrent fever attacks & Delphi 1 \\
Regulation of attacks & no \\
2. Constitutional symptoms & no \\
Actual criterion $^{\text {a }}$ & Delphi 1 \\
New criterion & no \\
3. Exclusion & Delphi 2 \\
Exclusion of autoinflammatory diseases & Delphi 1 \\
Exclusion of infections and related conditions & Delphi 2 \\
4. Age limitation for onset & Delphi 2 \\
5. Well-being between episodes & no \\
6. Growth and development & Delphi 2 \\
7. Response to steroid treatment & Delphi 2 \\
8. Other & Delphi 2 \\
\hline
\end{tabular}

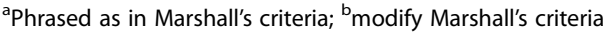

criteria, as well as the mandatory presence of at least one sign during each episode or even 2 in most episodes.

In the new criteria age at disease onset was modified in the new criteria at $<6$ years instead of $<5$ years, and more detailed description of other causes that clinician has to exclude were provided (other causes of recurrent fever, infection and other immunodeficiency than cyclic neutropenia). The new criteria specified that the pattern of growth have to be linear. After discussion, response to steroid was considered as supportive criteria and not included in the new criteria.

Both the newly proposed PFAPA criteria and the Marshall criteria were applied to a group of 80 PFAPA patients followed in Lausanne and Genoa in order to test their performance. The characteristics of patients included in the analysis are shown in Table 3. The proportion of boys and girls was 1:0.8. The median age at onset of PFAPA was 1.9 years old, the median age at diagnosis was 4 years.

All patients presented at least one of the 3 cardinal symptoms: aphthous stomatitis, pharyngitis, and cervical adenitis. Abdominal pain was the most frequently associated symptom, found in nearly $50 \%$ of the patients.

Only $41 / 80$ patients (51\%) fulfilled the new criteria. 31 patients were excluded because they didn't meet one of the classification criteria, 7 because of 2 criteria, and 1 because of 3 criteria. Application of both criterion 1 and 2 from the new criteria to our cohort resulted in the exclusion of 36/39 (92\%) patients.

Current modified Marshall criteria were met by $69 / 80$ patients (86\%). 7/80 didn't fulfill the Marshall criteria because of the age at onset (after 5 years of age).

By comparing patients excluded by the two sets of criteria, we noted that $6 / 80$ patients $(7,5 \%)$ were excluded 
Table 2 New set of PFAPA classification criteria and Modified Marshall's classification criteria with number of patients that didn't fulfill each criterion

\begin{tabular}{|c|c|c|c|}
\hline New classification criteria & Patients excluded $(\mathrm{N})$ & Modified Marshall's classification criteria & Patients excluded (N) \\
\hline $\begin{array}{l}\text { 1. Periodic Fever for at least } 6 \text { months: } \\
\text { a. Daily fever of at least } 38.5^{\circ} \mathrm{C} \text { (axillar) for } \\
2 \text { to } 7 \text { days } \\
\text { b. At least } 5 \text { regularly recurring fever episodes } \\
\text { with maximum of } 2 \text { months interval between them }\end{array}$ & 20 & $\begin{array}{l}\text { Regularly recurring fevers with an early } \\
\text { age onset }(<5 \text { years of age) }\end{array}$ & 7 \\
\hline $\begin{array}{l}\text { 2. Pharyngitis, cervical adenitis, oral aphthae: at least one } \\
\text { in every episode and at least } 2 \text { out of } 3 \text { in the majority } \\
\text { of episodes. }\end{array}$ & 21 & $\begin{array}{l}\text { 2. Constitutional symptoms in absence } \\
\text { of upper respiratory infection with at } \\
\text { least one of the following clinical signs: } \\
\text { - apthous stomatitis } \\
\text { - cervical lymphadenitis } \\
\text { - pharyngitis }\end{array}$ & 0 \\
\hline $\begin{array}{l}\text { 3. Exclusion of other causes of recurrent fever } \\
\text { (clinical or by laboratory depending on history) }\end{array}$ & 0 & 3. Exclusion of cyclic neutropenia & 0 \\
\hline $\begin{array}{l}\text { 4. Exclusion of infections, immunodeficiency and } \\
\text { cyclic neutropenia }\end{array}$ & 0 & & \\
\hline 5. Disease onset before the age of 6 years & 1 & & \\
\hline 6. Full recovery between episodes & 2 & $\begin{array}{l}\text { 4. Completely asymptomatic interval } \\
\text { between episodes }\end{array}$ & 2 \\
\hline 7. Normal linear growth & 4 & 5. Normal growth and development & 3 \\
\hline
\end{tabular}

Table 3 Characteristics of 80 PFAPA patients included in the analysis

\begin{tabular}{ll}
\hline Characteristic & \\
\hline M/F & 1.2 \\
Age at onset (years) & Median 1.9 (IQR 0.8-3.1) \\
Age at diagnosis (years) & Median 4 (IQR 2.6-6) \\
Pharyngitis & $88.7 \%$ \\
Adenitis & $85.0 \%$ \\
Aphthous Stomatitis & $71.2 \%$ \\
Headaches & $30.0 \%$ \\
Digestive: & \\
- Nausea & $6.25 \%$ \\
- Vomiting & $20.0 \%$ \\
- Abdominal pain & $48.7 \%$ \\
- Diarrhea & $22.5 \%$ \\
• Hepatomegaly & $1.2 \%$ \\
- Splenomegaly & $7.5 \%$ \\
Arthralgia & $33.7 \%$ \\
Arthritis & $1.2 \%$ \\
General malaise & $37.5 \%$ \\
Skin rash & $21.2 \%$ \\
Conjunctivitis & $8.7 \%$ \\
Myalgia & $26.2 \%$ \\
Neurological symptoms & $5 \%$ \\
Acute scrotum & \\
Peritonitis & $1.2 \%$ \\
\hline & $0 \%$ \\
\hline
\end{tabular}

from both. Table 2 provides the number of patients not satisfying each criterion for both sets.

\section{Discussion}

Thanks to a collaborative effort made by clinicians in the field of autoinflammatory diseases we developed a provisional new set of classification criteria for PFAPA disease based on experts' opinion and we tested their performance in a cohort of PFAPA patients. The new criteria seem quite difficult to apply, since they need a precise history that can't always be provided by parents. The characteristics of fever episodes provided in the new criteria are probably too restrictive and excluded nearly half of the patients. Conversely, the criterion "age at onset $<6$ years" seems better adapted and excluded only 1 patient [5]. There are not other substantial differences between new criteria and modified Marshall's criteria. Globally, the new criteria were able to classify fewer patients as PFAPA in respect to the modified Marshall's criteria if applied on our cohort of patients. By using these new criteria in clinical practice to diagnose PFAPA, a significant number of patients would not be diagnosed, and we consider them as not useful as diagnostic criteria. On the other hand, since they are very restrictive, these criteria probably would allow selecting a more homogeneous group of patients; therefore they may be suitable to select well-defined cohorts of patients for clinical research. In fact, Marshall criteria have been found to be poorly specific when tested in a population in which different monogenic periodic fevers are present [3]. This study presented some limitations and do not resolve the classification dilemma of PFAPA patients for 
different reasons. First of all, the elaboration of the new criteria was not the result of a statistical multivariate analysis of real patients with different confounding conditions (i.e. other autoinflammatory diseases, cyclic neutropenia or recurrent infections), but was made on the unique judgment of a panel of experts after the indications coming from a larger Delphi survey. The lack of a control group didn't allow us to calculate specificity and sensitivity of the new criteria. Moreover, the evaluation of the new criteria was done retrospectively on a cohort of only 80 patients with the diagnosis of PFAPA coming from only two centers, for which the diagnosis was based on the judgments of each center, not on the basis of a consensus among different experts.

This was the first attempt to create a new set of classification criteria for PFAPA syndrome based on a consensus among experts. With its limitations, this study allowed us to identify difficulties hidden in a consensus-based process, underlying the need for a definition of gold standard PFAPA patients to develop validated classification criteria, and paving the way for a better-structured project that is ongoing.

\section{Conclusions}

Our work led us to identify a new set of classification criteria for PFAPA syndrome that resulted to be too restrictive to be applied in daily clinical practice for the diagnosis of PFAPA. A new attempt, based on consensus among experts followed by evaluation and validation of the criteria on a cohort of gold standard patients is required, to define a set of classification criteria for PFAPA syndrome with a better performance in term of sensitivity and specificity.

\section{Abbreviation}

PFAPA: Periodic fever aphthous stomatitis pharyngitis cervical adenitis

\section{Acknowledgements}

The authors acknowledge Sivia Lapidus, Donato Rigante, and Joost Frenkel for their involvement in the Delphi process.

\begin{abstract}
Authors' contributions
$F V$, RC, equally contribute to the data collection, data analysis, and drafting the manuscript. SA an MC contribute do data colection and analysis. JA, SB, RB, PD, IKP, BN, SO, PP, SS, CW, MG and MH participate do Delphi process and to consensus conference. FV, RC, JA, SB, RB, PD, IKP, BN, SO, PP, SS, CW, $M G$ and $M H$ contribute to the final version of this paper. $M G$ and $M H$ conceived the study. All authors read and approved the final manuscript.
\end{abstract}

\section{Ethics approval and consent to participate}

The Ethics Committee approved this study. Due to the retrospective nature of this study, we obtained oral consent by at least one parent.

\section{Consent for publication}

Not applicable

\section{Competing interests}

The authors declare that they have no competing interests.

\section{Publisher's Note}

Springer Nature remains neutral with regard to jurisdictional claims in published maps and institutional affiliations.

\section{Author details}

${ }^{1}$ Istituto Pediatrico della Svizzera Italiana, Ospedale San Giovanni, 6500 Bellinzona, Switzerland. ${ }^{2}$ Unité Romande d'Immuno-rhumatologie Pédiatrique, CHUV, University of Lausanne, Lausanne and HUG, Geneva, Switzerland. ${ }^{3}$ Istituto Giannina Gaslini, Clinica Pediatrica e Reumatologia, PRINTO, Genoa, Italy. ${ }^{4}$ Pediatric Rheumatology, Hospital Sant Joan de Déu. Universitat de Barcelona, Esplugues de Llobregat, Barcelona, Spain.

${ }^{5}$ Department of Pediatrics, The Queen Silvia Children's Hospital, Goteborg, Sweden. 'Department of Pediatrics and Pediatric Rheumatology Service, Ruth Rappaport Children's Hospital, Rambam Medical Center, Haifa, Israel. ${ }^{7}$ Department of Paediatrics and Adolescent Medicine, Paediatric Rheumatology Unit, General University Hospital and 1st Faculty of Medicine, Charles University in Prague, Praha, Czech Republic. ${ }^{8}$ Rhumatologie pédiatrique, CHU Le Kremlin Bicêtre APHP, University of Paris Sud CEREMAIA, Paris, France. ${ }^{9}$ Unité d'Immunologie-Hématologie et Rhumatologie Pédiatriques CHU Paris - Hôpital Necker-Enfants Malades, Paris, France. ${ }^{10}$ Department of Pediatric Rheumatology, Hacettepe University, Ankara, Turkey. ${ }^{11}$ Service de pédiatrie médicale CHRU de Bordeaux, Bordeaux, France. ${ }^{12}$ Department of Infectious Diseases and Immunology, Children's Hospital, University of Munich, Munich, Germany. ${ }^{13}$ Department of Microbiology and Immunology, Laboratory Paediatric Immunology, UZ Leuven Hospital, Leuven, Belgium.

Received: 24 April 2018 Accepted: 13 September 2018

Published online: 21 September 2018

References

1. Marshall GS, Edwards KM, Butler J, Lawton AR. Syndrome of periodic fever, pharyngitis, and aphthous stomatitis. J Pediatr. 1987;110(1):43-6.

2. Thomas KT, Feder HM Jr, Lawton AR, Edwards KM. Periodic fever syndrome in children. J Pediatr. 1999;135(1):15-21.

3. Gattorno M, Caorsi R, Meini A, Cattalini M, Federici S, Zulian F, Cortis E, Calcagno G, Tommasini A, Consolini R, Simonini G, Pelagatti MA, Baldi M, Ceccherini I, Plebani A, Frenkel J, Sormani MP, Martini A. Differentiating PFAPA syndrome from monogenic periodic fevers. Pediatrics. 2009;124(4):e721-8.

4. Delbecq AL, Van de Ven AH, Gustafson DH. Group techniques for program planning. A guide to nominal group and Delphi processes. 1 ed. Glenview, ill, Scott, Foresman and company; 1975. Ruperto N, Meiorin S, Iusan SM, Ravelli a, Pistorio a, for the Paediatric rheumatology international trials organisation (PRINTO). Consensus procedures and their role in pediatric rheumatology. Curr Rheumatol Rep 2008; 10(2):142-146.

5. Hofer M, Pillet P, Cochard M-M, Berg S, Krol P, Kone-Paut I, Rigante D, Hentgen V, Anton J, Brik R, Neven B, Touitou I, Kaiser D, Duquesne A, Wouters C, Gattorno M. International periodic fever, aphthous stomatitis, pharyngitis, cervical adenitis syndrome cohort: description of distinct phenotypes in 301 patients. Rheumatology (Oxford). 2014;53(6):1125-9.

\footnotetext{
Ready to submit your research? Choose BMC and benefit from:

- fast, convenient online submission

- thorough peer review by experienced researchers in your field

- rapid publication on acceptance

- support for research data, including large and complex data types

- gold Open Access which fosters wider collaboration and increased citations

- maximum visibility for your research: over $100 \mathrm{M}$ website views per year

At BMC, research is always in progress.

Learn more biomedcentral.com/submissions
} 